the British Ministry of Aviation among them-who predicted the demise of manned aircraft ten years ago. The FOBS system consists of a launcher 113 feet long, with six first stage rockets, launching a rocket into an orbit about 100 miles from the Earth. Before the first orbit is complete, the bomb is brought out of orbit on to the target. According to Jane's, observations at Farnborough of tests of the FOBS system suggest that the bomb is about 6 feet 6 inches long and 4 feet in diameter.

In the aircraft sphere, there have been several disappointments. Variable geometry has had a bad year, with the disappointing performance of the F111, and the abandonment of the ambitious design for the American Supersonic Transport in favour of a fixed wing design. Concorde still remains on the ground. But the British vertical take-off fighter, the Harrier, did come into service with the RAF, and this may turn out to have been an event of considerable significance. Mr Taylor (who makes all these points) is not alone in predicting that the Harrier will be as successful in export markets as the Hunter was.

As for Jane's itself, there are few changes in this year's edition. For the first time the missiles section is divided into two, with military missiles separated from space research vehicles. Airships are back in favour, although there are only four of them, one a replica put together for the purposes of a film. Many aircraft are listed of which only one prototype has ever been built, often by single enthusiasts to their own designs. This kind of thoroughness is typical of Jane's, which remains the standard work about aeroplanes. NIGEL HAwkes

\section{SIMULATION AND ANALYSIS}

\section{Mathematical Models for the Evaluation of Electric and Magnetic Fields}

By O. V. Tozoni. Edited by A. A. Kaye. Translated by Seripta Technica. Pp. 328. (Iliffe: London, 1968.) 75s.

THIs book is a contribution to the growing literature dealing with computational methods for solving engineering problems in electromagnetism. The methods in this book are restricted mainly to finding solutions of Laplace's equation and Poisson's equation, though one or two sections are concerned with non-linear equations; at least two-thirds of the book is devoted to two-dimensional fields.

Most space is allotted to finding the fiold when the potential (or its normal derivative) is prescribed on a complicated boundary so that an analytical solution is completely out of the question. There are three ways of obtaining an answer: by analogue computer, by digital computer, or by a mixture of computation and analysis. The author dismisses rather rapidly the digital computer in spite of the occasional section later in the book. This dismissal may reflect the capacity of the computers available in Russia ten years ago (the references in the original text cease at 1960) and the author might write differently today. Certainly I would seriously consider using an integral equation approach together with a digital computer before embarking on some of the simulation described.

The author's main aim is to describe a combination of simulation and analysis which has proved to be practically useful. In this respect engineers will find it a helpful reference because of the careful explanation and the attention paid to estimating the errors likely to arise in the actual simulation; however, there are no exercises for students to try. The analytical methods are described in some detail and so is the simulation apparatus; the author points out the practical difficulties involved in the con. struction of such apparatus and also notes why these difficulties may make one form of simulation more acceptable than another.
The first chapter goes over the usual properties of an analytical function of a complex variable and includes a derivation $a b$ initio of Poisson's integral representation. Illustrations of conformal mapping and the use of the Poisson representation occupy the later sections of the chapter. Those who have taken a standard university course in mathematics for engineers should be able to follow this chapter and, indeed, most of the analysis in the book.

The second chapter is concerned with simulating the conformal mapping of an arbitrary shape into a standard shape such as a strip. The simulation is achieved by means of a current flow in a thin sheet and one noteworthy illustration is that for the Schwartz-Christoffel transformation.

In the third chapter the method is applied to the determination of the magnetic field in a direct current machine, and a four pole machine with gaps between the poles and yoke is examined in some detail.

The fourth chapter takes up the problems of Poisson's equation and of domains which extend to infinity. The author shows, by an ingenious mapping of part of the region, how a finite analogue can be constructed for the second problem with an error which lies within acceptable limits. As regards the first problem, there is a full discussion of the errors introduced by the simulation of the sources in Poisson's equation; the imperfections of the device used for Dirichlet's problem are also treated fully. The last two sections cover non-linear media and the design of three-phase lines.

Three-dimensional fields are considered in the fifth chapter. The main problem that is discussed is the simulation of stray and cathode currents in underground pipes and electric railways.

The standard of translation is reasonably good, although there are one or two lapses- to say that a Riemann surface disintegrates when branch lines are chosen is not a happy choice of phrase. But this is a minor criticism of a book which is a worthwhile addition to the field of analogue computation.

D. S. JoNes

\section{PROGRAMMING TEXT}

\section{Recursive Techniques in Programming}

By D. W. Barron. (Macdonald Computer Monographs.) Pp. 64. (Macdonald: London, 1968.) 25s.

A GOOD test of one's sympathy with a textbook is the extent to which one feels urged to incorporate the contents into one's teaching. I regard this as an excellent little book. I think the author has done a wonderful job in introducing so many facets of the subject so concisely and I shall use much of the material.

It is valuable to have a clearly illustrated description of a fundamental difference between ALGOL and FORTRAN in a context in which the differences are not easy to describe. The inclusion of description of recursive systems in list processing languagos is also very wolcome. It is a pity that the facilities of PL/1 are dismissed so lightly and, of course, there is no mention of the advances in ALGOL 68. This dates the book a little after only a single year since publication.

There is a very comprehensive range of recursive applications in mathematics, sorting, compiler writing, meta language definitions and so on.

The chapter on the mechanics of recursion introduces both hardware and software techniques. The concepts and mechanism of stacks are very well described and many will find the clear description of much bewildering jargon a great boon.

This book is a "must" for a professional programmer and will be of educational value to a much wider class of reader. 